\title{
VARIATIONAL MUTI-STEPS METHOD TO SOLVE DAMPED OSCILLATION EQUATION
}

\author{
Riza Ibnu A*, Adhi R., Susilawati \\ Department of Informatics, Computer Science, Universitas Singaperbangsa Karawang, \\ Jl. HS Ronggowaluyo, Telukjambe Timur, Karawang 41361
}

Recieved: $12^{\text {th }}$ September 2017; Revised: $20^{\text {th }}$ October 2017; Accepted: $26^{\text {th }}$ October 2017

\begin{abstract}
This paper aims to identifying the numerical method accuracy of the analytical solution of the damped oscillation equation motion. Adams method of 4th order, Milne method and Adams-Simpson method are used to find numerical solutions. Value of $y(1), y(2), y(3)$ obtained from The 4th order Runge-Kutta method. They used as initial value of multistep method. Then, the numerical solution result was compared with analytical solution. From the research result, it is found that 4th order Adams method has the best accuracy.
\end{abstract}

Keywords: $4^{\text {th }}$ order Adams method; Milne method; Adams-Simpson method; 4th order Runge-Kutta method

\section{Introduction}

Oscillation is a move back and forth around the equilibrium position. Many oscillatory phenomena can be observed in everyday life. Leaf movement on the tree trunk, vibration strings of the guitar, and springing motion because there is a load, there are example of an oscillation phenomenon. Oscillation is very important in the development of computers, mobile phones, and other digital equipment. Without oscillation, the electrical charge on the mobile phone antenna will not work. ${ }^{1}$

In fact, There are forces that cause vibration also there are force that inhibits vibration motion. So that all vibration motion eventually decreases its energy and stops vibrating. Mathematically the oscillation motion is modeled by the 2nd order differential equation.

The solution of differential equations can be solved by analytic and numerical methods. The analytic method will produce a true solution that is usually difficult to find the solution. While numerical methods produce approximation solution that can be done directly without simplification of physical

*Corresponding author.

E-Mail: rizaibnuadam@gmail.com parameters, but requires a standard trial function determined by analytical methods. In previous research, numerical modeling has been done, to predict oscillation motion on vibration sensors based on electromagnetic induction. $^{2}$ The results obtained that the numerical method is able to model the oscillation motion without having to know the analytical solution first.

There are many methods that can be used to solve differential equations, one of which is the multi steps method. The multi steps method is a method that requires two or more previous values to obtain the present value. These initial values are derived from the onestep method (Euler or Runge-Kutta method).

The multi-step method is called the predictor-corrector method, because in the solution used predictor equations and corrector equations. The Adams method, Milne method and Adams-Simpson method are many step methods. Previous research has shown that numerical calculations use the Adams method. Milne method and AdamsSimpson method is more accurate than Euler or Runge-Kutta method. ${ }^{3,4,5}$

In this research, we determine analytical solution of solution of second-order 
differential equation which arise in problem of damped oscillation motion. The analytical solution was compared to analyze the accuracy of numerical solutions of the Adams method, the Milne method and Adams-Simpson method. The one-step method used to find the initial solution is the fourth-order Runge-Kutta method. The Runge-Kutta method is an alternative to the Taylor series method that does not require derived counts. The advantages of this Runge-Kutta method have higher accuracy than the Euler method, Heun method and Taylor series method.

\section{Methods}

The initial step of this research is to determine the initial equation of damped oscillation motion which is then solved analytically. Equation (1) represents the equation of damped oscillation motion.

$$
\frac{d^{2} y}{d t^{2}}+\frac{b}{m} \frac{d y}{d t}+\frac{k}{m} y=0
$$

Equation (1) can be written analytically as equation (2)

$$
y=A e^{-b / 2 m \cos \omega^{\prime} t},
$$

with

$$
\omega^{\prime}=\sqrt{\frac{k}{m}-\left(\frac{b}{2 m}\right)^{2}} .
$$

Based on equation (1) and (2) $y, t, b, k, m, A$, dan $\omega^{\prime}$ are position $(\mathrm{m})$, time (s) damping constant $(\mathrm{Ns} / \mathrm{m})$, elasticity constant $(\mathrm{N} / \mathrm{M})$, mass (Kg), amplitude (m), and corner frequency $(\mathrm{Hz})$ respectively. ${ }^{6}$

\section{4th order Runge-Kutta Method}

In this step, equation (1) was resolved numerically by using RK4. Equation (1) is 2nd order differential equation. To simplify the calculation process, then we are using analogy, which is $d^{2} y / d t^{2}=y^{\prime \prime}=z^{\prime}$ and $d y / d t=y^{\prime}=z$, so equation (3a) and (3b) obtained.

$$
y^{\prime}=z
$$

$$
z^{\prime}=-\frac{k}{m} y-\frac{b}{m} z
$$

Based on RK4 algorithm, two solutions of differential equation (4a and 4b) are obtained.

$$
\begin{gathered}
p 1=h \times z(i), \\
p 2=h \times\left(z(i)+\frac{1}{2} q 1\right), \\
p 3=h \times\left(z(i)+\frac{1}{2} q 2\right), \\
p 4=h \times(z(i)+q 3), \\
y(i+1)=y(i)+\left[\frac{1}{6}(p 1+p 2+p 3+p 4)\right]
\end{gathered}
$$

and

$$
\begin{gathered}
q 1=h \times\left(-\frac{k}{m} y(i)-\frac{b}{m} z(i)\right), \\
q 2=h \times\left[-\frac{k}{m}\left(y(i)+\frac{1}{2} p 1\right)-\right. \\
\left.\frac{b}{m}\left(z(i)+\frac{1}{2} q 1\right)\right], \\
q 3=h \times\left[-\frac{k}{m}\left(y(i)+\frac{1}{2} p 2\right)-\right. \\
\left.\frac{b}{m}\left(z(i)+\frac{1}{2} q 2\right)\right], \\
q 4=h \times\left[-\frac{k}{m}(y(i)+p 3)-\frac{b}{m}(z(i)+\right. \\
q 3)], \\
z(i+1)=z(i)+\left[\frac{1}{6}(q 1+q 2+q 3+q 4)\right]
\end{gathered}
$$

where $h$ is the range of each iteration.

\section{4th order Adams Methods}

After the calculation with RK4 has done, we solved equation (1) using Adams4. The solution obtained by RK4, then substituted into Adam4 as initial value. Based on Adams4 algorithm we obtained equation as equation (5a) and (5b) 


$$
\begin{gathered}
y(i+1)=y(i)+\left[\frac { h } { 2 4 } \left(55 y^{\prime}(i)-\right.\right. \\
\left.\left.59 y^{\prime}(i-1)+37 y^{\prime}(i-2)-9 y^{\prime}(i-3)\right)\right]
\end{gathered}
$$

and

$$
\begin{gathered}
z(i+1)=z(i)+\left[\frac { h } { 2 4 } \left(55 z^{\prime}(i)-\right.\right. \\
\left.\left.59 z^{\prime}(i-1)+37 z^{\prime}(i-2)-9 z^{\prime}(i-3)\right)\right]
\end{gathered}
$$

\section{Milne Method}

Milne method is similar to Adams4. This method requires the initial value that generated from RK4. Equation (1) was solved by the Milne algorithm so that the equation (6a) and (6b) is obtained.

$$
\begin{gathered}
y(i+1)=y(i-3)+\left[\frac { 4 h } { 3 } \left(2 y^{\prime}(i)-\right.\right. \\
\left.\left.y^{\prime}(i-1)+2 y^{\prime}(i-2)\right)\right]
\end{gathered}
$$

and

$$
\begin{aligned}
& z(i+1)=z(i-3)+\left[\frac { 4 h } { 3 } \left(2 z^{\prime}(i)-\right.\right. \\
& \left.\left.z^{\prime}(i-1)+2 z^{\prime}(i-2)\right)\right]
\end{aligned}
$$

\section{Adams-Simpson Method}

Like Adam4 and Milne, AS requires the initial value to calculating. However AS predicts the value to be searched with the predictor equation, after which the predicted value will be corrected by the corrector equation. Based on the AS method algorithm, so equation predictor is

$$
\begin{aligned}
& y(i+1)=y(i)+\left[\frac { h } { 2 } \left(3 y^{\prime}(i)-\right.\right. \\
& \left.\left.3 y^{\prime}(i-1)\right)\right]
\end{aligned}
$$

and

$$
z(i+1)=z(i)+\left[\frac{h}{2}\left(3 z^{\prime}(i)-3 z^{\prime}(i-1)\right)\right]
$$

and then the corrector solution is

$$
\begin{array}{r}
y(i+1)=y(i-1)+\left[\frac { h } { 3 } \left(3 y^{\prime}(i+1)+\right.\right. \\
\left.\left.4 y^{\prime}(i)-y^{\prime}(i-1)\right)\right](8 \mathrm{a})
\end{array}
$$

and

$$
\begin{array}{r}
z(i+1)=z(i-1)+\left[\frac { h } { 3 } \left(3 z^{\prime}(i+1)+\right.\right. \\
\left.\left.4 z^{\prime}(i)-z^{\prime}(i-1)\right)\right](8 \mathrm{~b})
\end{array}
$$

\section{Result and Discussion}

Research about numerical solutions of damped oscillation motion equation has finished. The solution is obtained by modeling equation (2). Penelitian mengenai solusi numeric dari persamaan gerak osilasi teredam dilakukan dengan memodelkan persamaan 2. Modelling using RK4, Adam4, Milne and AS algorithms. The initial value $b$, $k, m$, and $A$ is $0.25 \mathrm{Ns} / \mathrm{m}, 3 \mathrm{~N} / \mathrm{m}, 0.5 \mathrm{Kg}$, and $1 \mathrm{~m}$ respectively. Where initial position $y(0)=A$, and $h=0.1$ with $t=10 \mathrm{~s}$. The value $y(n)$ with $n=0,1,2,3$, has been obtained from RK4, for substituted into Adam4, Milne and AS as initial values. The modeling results are shown in Figure 1.

From Figure 1 it is known that the analytical solution is represented by a black cross. The analytical solution describes the damped oscillation motion where the initial drift is given a value of $1 \mathrm{~m}$ which then progressively stops by 10 seconds. The same is true of numerical solutions generated by Adams4 and RK4. The Adams4 solution is indicated by a red circle marker while RK4 is indicated by a purple star. In Adam 4 it is known that the numerical solutions generated each iteration are very close to analytical solutions. However in RK4, although the resulting solution appears to follow the same pattern with the analytical solution, but the results have different values. It is seen from the 1st to 6th seconds. Different conditions are shown by Mine and AS, where both methods have an enlarged deviation. The Milne method shown by the green box marks has been seen to be inconsistent since the 1st second. The same is followed by the AS indicated by the blue plus sign. However, the deviation produced by the AS is smaller than Milne. 


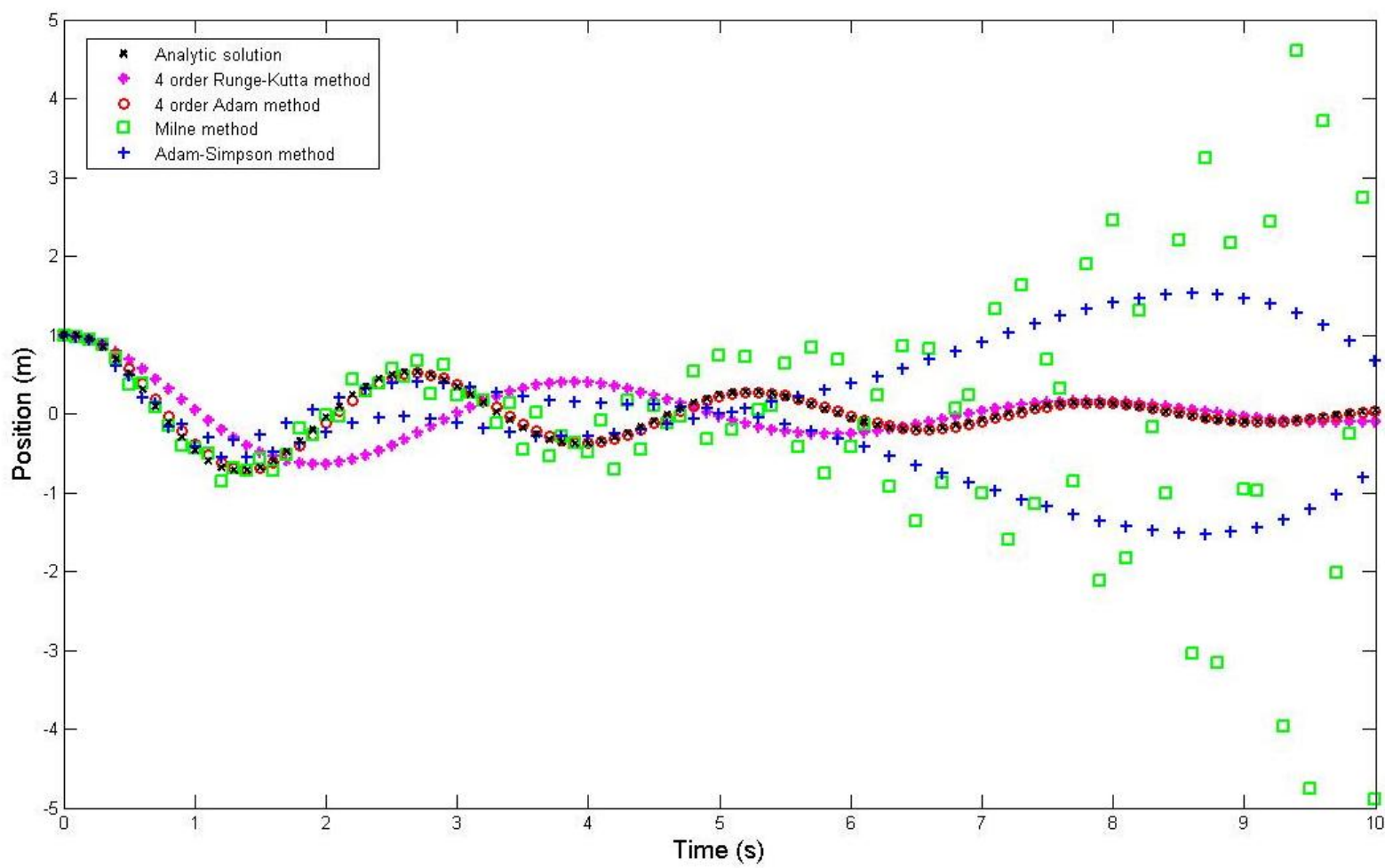

Figure 1. Relationship of position to time using analytical solutions, RK4, Adam4, Milne and AS

\section{Conclusion}

Based on the results of the analysis, it can be seen that the numerical solution generated by Adams4 has a very good accuracy compared to numerical solutions of the other three methods. Milne became the worst method of accuracy, as was the AS method. Although the AS does a more complicated calculation because of the predictor and corrector equations, it does not make this method the best. On the other hand RK4 emerged as the most efficient method because it does not require many calculation processes such as multi-step method, but the accuracy produced better than Milne and AS

\section{Acknowledgment}

We are partly supported by the ResearchCluster Grant Program of the Universitas Singaperbangsa Karawang.

\section{References}

1. Abdullah M. Basic Physics I. Bandung: ITB; 2016. (in Indonesia)

2. Adam R I. Newton-Raphson and Euler Methods Merger to Solving Motion Equation on The Magnetic Oscillator. Jurnal Pendidikan Fisika Dan Keilmuan (JPFK). 2017 March; 3(1): 13-18. (in Indonesia)

3. Yang X, Ralescu D A. Adams Method for Solving Uncertain Differential Equations. Applied Mathematics and Computation. 2015; 270: 993-1003.

4. Gao R. Milne Method for Solving Uncertain Differential Equations. Applied Mathematics and Computation. 2016; 274: 774-785.

5. Wang X, Ning Y, Moughal T A, Chen X. Adams-Simpson Method for Solving Uncertain Differential Equations. Applied Mathematics and Computation. 2015; 271: 209-219.

6. Serway R A, Jewett J W. Physichs for Scientiest and Engineers (6th ed). USA : Thomson; 2004. 